\title{
Preoperative MRI is helpful but not sufficient to detect associated lesions in patients with chronic ankle instability
}

\author{
Kevin Staats ${ }^{1}$ - Manuel Sabeti-Aschraf ${ }^{1} \cdot$ Sebastian Apprich $^{1} \cdot$ Hannes Platzgummer $^{2}$. \\ Stephan E. Puchner ${ }^{1} \cdot$ Johannes Holinka $^{1} \cdot$ Reinhard Windhager $^{1} \cdot$ Reinhard Schuh $^{1}$
}

Received: 17 October 2016 / Accepted: 3 May 2017 / Published online: 15 May 2017

(C) The Author(s) 2017. This article is an open access publication

\begin{abstract}
Purpose The aim of this study was to determine the reliability and validity of preoperative magnetic resonance imaging (MRI) scans for the detection of additional pathologies in patients with chronic ankle instability (CAI) compared to arthroscopic findings.

Methods Preoperative MRI images of 30 patients were evaluated regarding articular and periarticular comorbidities and compared to intraoperative findings. The reliability of MRI was determined by calculating specificity, sensitivity, as well as positive and negative predictive values. The accuracy of the classification of cartilage lesions by Outerbridge and Berndt and Harty rating scales was determined by calculating the area under the receiver operating curve (AUC).

Results In total, 72 additional pathologies were found arthroscopically compared to 73 lesions gathered from MRI images. Sensitivity ranged from $89 \%$ for peroneal tendinopathy to $28 \%$ for additional ligamentous lesions. Specificity ranged from $100 \%$ for anterolateral impingement, loose bodies and peroneal tendinopathy to $38 \%$ for additional ligamentous lesions. For cartilage lesions, sensitivity was at $91 \%$ and specificity was at $55 \%$ for the Outerbridge grading scale. For the Berndt and Harty classification system, sensitivity was at $91 \%$ and specificity was at $28 \%$. Correlation of additional pathologies ranged from
\end{abstract}

Reinhard Schuh

reinhard.schuh@meduniwien.ac.at

1 Department of Orthopaedic Surgery, Medical University of Vienna, Waehringer Guertel 18-20, 1090 Vienna, Austria

2 Department of Biomedical Imaging and Image-guided Therapy, Medical University of Vienna, Waehringer Guertel 18-20, 1090 Vienna, Austria weak $\left(r_{\mathrm{s}}=0.48 ; p=0.02\right)$ to moderate results $\left(r_{\mathrm{s}}=0.67\right.$; $p<0.001)$.

Conclusion CAI is associated with a high incidence of additional pathologies. In some cases, MRI delivers insufficient results, which may lead to misinterpretation of present comorbidities. MRI is a helpful tool for preoperative evaluation, but arthroscopy remains gold standard in the diagnosis of associated lesions in patients with CAI. Level of evidence III.

Keywords Ankle $\cdot$ Instability $\cdot$ Arthroscopy $\cdot$ MRI

\section{Introduction}

Lateral ankle sprains are one of the most common injuries caused by stressful inversion [8, 24, 26]. Despite the fact that most patients can be treated conservatively with success, approximately $30 \%$ of the patients with ankle sprains remain with chronic lateral ankle instability (CAI) [12]. With recurrent sprains and persistent pain, a subset of CAI patients requires surgical treatment due to a mechanical dysfunction of the lateral ligamentous complex [3, 15]. Surgical treatment aims for anatomical repair [24]. This can be achieved arthroscopically or through an open approach by transosseous fixation of the torn ligamentous fibres to the anteroinferior aspect of the fibula [20]. Additionally, the inferior extensor retinaculum can be augmented to the fibular periosteum [5].

Isolated injuries of the lateral ligamentous complex are scarce and additional lesions are commonly investigated in CAI [16]. In many cases, this is due to pathomechanical forces acting on the unstable ankle [18]. Peroneal tendon pathologies, concomitant ligamentous and syndesmotic injuries, anterolateral impingement or osteochondral 
lesions are the most common associated injuries [8, 23]. Although magnetic resonance imaging (MRI) represents a standard diagnostic procedure, information about reliability and validity remains inconclusive $[4,21,27]$. Therefore, it is still debated whether MRI delivers exact and reliable preoperative information about the extent involved injured structures [14]. Due to the advantage of direct visualization of intraarticular structures, arthroscopy is considered as gold standard [4, 10, 11, 21, 25].

The aim of this study was to determine the reliability and validity of preoperative MRI scans for the detection of additional bony, ligamentous, musculous and soft tissue-related pathologies in patients with CAI compared to arthroscopic findings. We hypothesized that compared to arthroscopic findings, preoperative MRI may lead to misinterpretation of the extent and the amount of additional pathologies in patients with CAI. Literature about the evaluation of additional pathologies in CAI is scarce and most authors only focused on certain pathologies. This present study should give the reader an insight on the reliability of preoperative MRI of CAI for the most common additional pathologies combined.

\section{Materials and methods}

Thirty patients with CAI and failed conservative treatment were included in this retrospective investigation. All patients presented with recurrent ankle sprains in their medical history. Physical examination was performed by two orthopaedic surgeons (RS and MS) with years of experience in foot and ankle surgery. CAI was suspected in patients with positive anterior drawer test and/or positive talar tilt test. CAI was subsequently diagnosed if MRI revealed rupture or partial rupture of the anterior talofibular ligament (ATFL). All patients received an anatomical reconstruction with suture anchor technique between February 2012 and January 2016. Table 1 summarizes the demographic data of all patients included.

\section{MR imaging and analysis}

Preoperative MRI examination was performed using a $3 \mathrm{~T}$ scanner (Siemens Magnetom Trio, A Tim System; gradient strength of $40 \mathrm{mT} / \mathrm{m}$ ) with a dedicated 8-channel foot/ ankle coil. Patients were placed in feet-first supine position with the ankle joint in the centre of the scanner. A standard native morphological MRI protocol containing the following sequence was used: (1) An axial T2-weighted (T2-w) turbo spin echo (TSE) sequence with fat suppression (fs) (repetition time (TR) $5520 \mathrm{~ms}$, echo time (TE) $118 \mathrm{~ms}$, bandwidth (BW) 203, field of view (FoV) $150 \times 150$, matrix $346 \times 364$, slice thickness (SL) $3 \mathrm{~mm}$, acquisition
Table 1 Demographic data of all patients involved

\begin{tabular}{ll}
\hline Variable & Data \\
\hline Age, years & $39.1 \pm 15.1(18-71)$ \\
Sex & Male $n=15 /$ female $n=15$ \\
Side involved & Left $n=14 /$ right $n=16$ \\
Symptom duration, weeks & $28.4 \pm 12.7(13-49)$ \\
$\begin{array}{l}\text { Time between MRI and arthroscopy, } \\
\text { months }\end{array}$ & $3.9 \pm 3.5(0-15)$ \\
\hline
\end{tabular}

Data are shown as mean with \pm standard deviation and (range) unless otherwise indicated

time (TA) 02:57 min) (2) a sagittal T1-w turbo inversion recovery magnitude (TIRM) sequence (inversion recovery $220 \mathrm{~ms}$, TR $6000 \mathrm{~ms}$, TE $51 \mathrm{~ms}$, BW 252, FoV $180 \times 180$, matrix $256 \times 320$, SL $3 \mathrm{~mm}$, TA 04:25 $\mathrm{min}$ ), (3) a sagittal T1-w spin echo (SE) sequence (TR $548 \mathrm{~ms}$, TE $16 \mathrm{~ms}$, BW 203, FoV $180 \times 180$, matrix $288 \times 384$, SL $3 \mathrm{~mm}$, TA 05:55 $\mathrm{min}$ ), (4) an axial proton density-weighted (PD-w) spectral attenuated inversion recovery (SPAIR) sequence (TR $3500 \mathrm{~ms}$, TE $31 \mathrm{~ms}$, BW 191, FoV $180 \times 180$, matrix $240 \times 320$, SL $3 \mathrm{~mm}$, TA 03:06 $\mathrm{min}$ ) and (5) a coronal PD-w SPAIR sequence (TR $5100 \mathrm{~ms}$, TE $48 \mathrm{~ms}$, BW 352 , FoV $200 \times 200$, matrix $308 \times 384$, SL $3 \mathrm{~mm}$, TA 06:08 $\mathrm{min}$ ). Total scan time including a set of localizers was approximately $24 \mathrm{~min}$.

All images were reviewed independently by one orthopaedic resident (KS) with special interest in MRI and ankle surgery, including monitoring dissections of anatomic specimens and previous training in MRI of the ankle joint, and one reader with years of experience in foot and ankle surgery (RS) on a picture archiving and communication system (PACS) workstation. The readers were blinded to the patient's medical history and arthroscopic findings. Ligamentous lesions were diagnosed using the following criteria described by Joshy et al. [14]: discontinuity, curved contour, non-visualization or signal enhancement within the ligament. Cartilage lesions found during arthroscopy were evaluated using the Outerbridge grading scale [22]. Arthroscopically observed cartilage lesions were compared to preoperative MRI images by using the classification system published by Berndt and Harty $(\mathrm{B}+\mathrm{H})$ and Outerbridge [2]. Additional comorbidities like anterolateral impingement, other ligamentous and syndesmotic pathologies, bony spurs, peroneal tendinopathy and loose bodies found in the MRI-based images were evaluated and detected using the criteria published by Linklater [19] and Alparslan et al. [1]. Hypertrophic synovium and fibrosis causing anterolateral impingement was characterized on MRI as an abnormal soft tissue mass in the anterolateral gutter showing low T1- and low to intermediate T2-signals [13]. 


\section{Surgical technique}

All patients underwent anatomical lateral ligament reconstruction with suture anchor technique. A $2.7-\mathrm{mm}$ arthroscope and standard anteromedial and anterolateral portals were used. Prior to the reconstructive procedure, a full diagnostic arthroscopy with adequate documentation was performed. Pathologies of the lateral ligament complex were confirmed under direct visualization with a forceful inversion manoeuvre. A ligamentous pathology was diagnosed when a non-anatomical/abnormal course of the ligament, a decreased tensity or discontinuity of the ligament were detectable. Bony spurs and other tissue causing potential impingement as well as potential loose bodies were removed extensively. For mini-open ligament repair resorbable suture anchors were used (BioMini-Suture-Tak ${ }^{\circledR}$, Arthrex).

\section{Intraoperative evaluation}

All arthroscopic findings were retrospectively reviewed by an experienced orthopaedic surgeon (RS) and a trained assessor (KS) independently. Again the readers were blinded to the patient's name and medical history. Surgical records and intraoperative photographic documentation were examined considering ligamentous pathologies, osteochondral lesions by using the Outerbridge grading system, the presence of anterolateral impingement, loose bodies, bony spurs and peroneal tendinopathy.

This study was approved by the local ethics committee of the Medical University of Vienna (EK No. 1072/2016).

\section{Statistical evaluation}

The frequency of pathologic findings from preoperative MRI scans and intraoperative documentation are compiled to calculate sensitivity, specificity, positive predictive value (PPV), negative predictive value (NPV) with lower and upper $95 \%$ confidence interval (CI) levels. Accuracy for the detection of cartilage damage with both classification systems (Outerbridge and $\mathrm{B}+\mathrm{H}$ ) is determined by calculating the area under the receiver operating curve (with 95\% CI).
For the evaluation of a potential correlation between radiologic and arthroscopic features, Spearman's rank order correlation was used and expressed as $r$ values $\left(r_{\mathrm{s}}\right)$. Arthroscopic findings were accounted for standard of reference. Interrater and intrarater reliability was assessed by comparing the independent results of two evaluation cycles. The readers were able to reproduce their own findings 4 weeks apart. The agreement between the two readers was excellent to good, depending on the comorbidity and modality. Table 2 displays the inter- and intrarater reliability of the additional lesions found in MRI and arthroscopy. Basic descriptive statistics were performed using SPSS software version 23 (SPSS Inc., Chicago, USA), and $p$ values $<0.05$ were considered as statistical significant.

In addition, a post hoc sample size calculation indicated we had 0.81 power to detect an effect size of 0.47 , with alpha set at 0.05. Sample size was calculated using free sample size calculating software $G$ *Power version 3.1.9.2 (Franz, Universitat Kiel, Germany).

\section{Results}

\section{Arthroscopic and MRI-based findings}

All patients $(n=30)$ showed changes (total rupture: $n=16$, partial rupture: $n=6$, elongation: $n=8$ ) both in MRI and arthroscopically in the course of the ATFL, with all of these requiring surgical treatment in terms of an anatomical ligament repair using suture anchors for refixation.

Overall, 72 additional pathologies arthroscopically compared to 73 lesions in MRI images were found in this study. A per-patient analysis illustrated that each individual showed an average of two comorbidities intraoperatively (range 1-4) and radiologically (0-6). Figure 1 and Table 3 summarize all additional pathologies found during arthroscopy and in preoperative MRI scans.

In $43 \%(n=13)$ of all patients, additional ligament pathologies were documented intraoperatively, whereas in $33 \%(n=10)$ preoperative MRI revealed an additional ligament lesion. Details on additional ligament pathologies are shown in Table 4.

Table 2 Inter- and intrarater reliability of MRI and arthroscopic findings

\begin{tabular}{lllll}
\hline & Interrater MRI & Interrater arthroscopy & Intrarater MRI & Intrarater arthroscopy \\
\hline Anterolateral impingement & 0.851 & 0.998 & 0.949 & 1 \\
Anterior tibial bony spur & 0.833 & 0.969 & 0.903 & 0.976 \\
Peroneal tendinopathy & 0.734 & 0.886 & 0.884 & 0.982 \\
Loose bodies & 0.737 & 0.987 & 0.992 & 1 \\
Additional ligament lesions & 0.702 & 0.823 & 0.792 & 1 \\
\hline
\end{tabular}

Inter- and intrarater reliability was assessed by comparing the results of two independent readers two evaluation cycles 4 weeks apart 


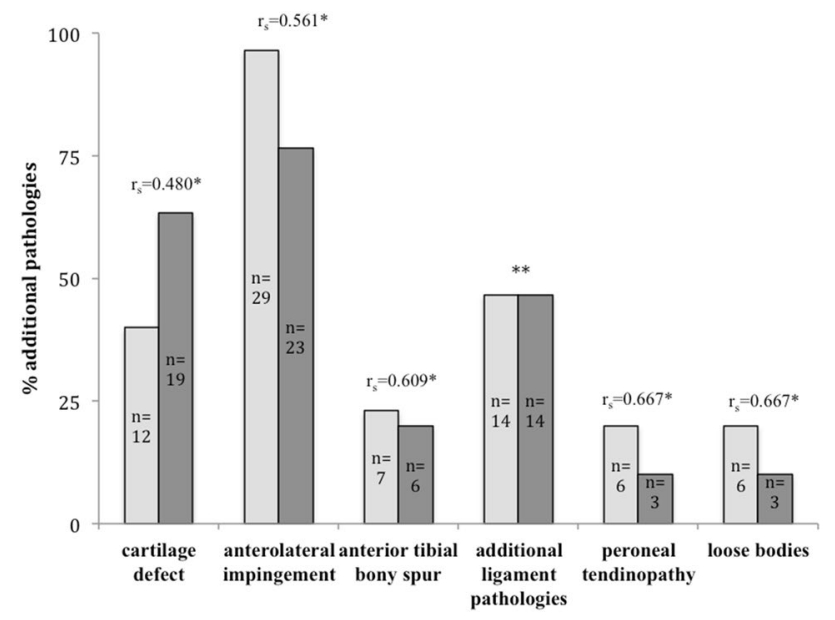

Fig. 1 Prevalence and correlation of additional lesions in CAI patients. Lesions were detected intraoperatively (bright grey) and in MRI (dark grey). Correlation was determined by calculating Spearman's rank coefficient $\left(r_{\mathrm{s}}\right) ;\left({ }^{*}\right.$ statistically significant), **correlation not calculated because of misleading results due to different entities found in MRI and arthroscopy (Table 3)

\section{Correlation between preoperative MRI and arthroscopic findings}

Correlation analysis for additional pathologies revealed low to moderate results with statistical significance (Fig. 1). An overall analysis of the numbers of pathologies evaluated for each patient from MRI and arthroscopy showed a weak but significant correlation $\left(r_{\mathrm{s}}=0.41 ; p=0.025\right)$.

Evaluation of cartilage damage in MRI and arthroscopy showed moderate results with high statistical significance for Outerbridge and poor to moderate correlation with statistical significance for $\mathrm{B}+\mathrm{H}$ classification systems (Tables 5, 6).

\section{Sensitivity and specificity of preoperative MRI scans regarding additional cartilage injuries in CAI}

Table 6 gives a detailed summary of the results considering sensitivity and specificity. Accuracy for the Outerbridge classification system determined by the area under
Table 3 Reliability of MRI for additional pathologies

\begin{tabular}{lllll}
\hline & Sensitivity & Specificity & PPV & NPV \\
\hline Anterolateral impingement & $79.3 \%$ & $100 \%$ & $100 \%$ & $14.3 \%$ \\
& $($ CI 60-91) & (CI 5-100) & (CI 82-100) & (CI 0-58) \\
Anterior tibial bony spurs & $57.1 \%$ & $91.3 \%$ & $66.3 \%$ & $87.5 \%$ \\
& $($ CI 20-88) & (CI 70-98) & (CI 24-94) & (CI 67-97) \\
Peroneal tendinopathy & $89.0 \%$ & $100 \%$ & $50.2 \%$ & $100 \%$ \\
& $($ CI 70-97) & (CI 31-100) & (CI 14-86) & (CI 83-100) \\
Loose bodies & $33.3 \%$ & $100 \%$ & $100 \%$ & $80.1 \%$ \\
& $($ CI 9-69) & (CI 83-100) & (CI 31-100) & (CI 61-92) \\
\hline
\end{tabular}

Reliability was evaluated by calculating sensitivity, specificity, positive predictive value $(P P V)$ and negative predictive value $(N P V)$. All values are shown with $95 \%$ confidence intervals $(C I)$

\begin{tabular}{|c|c|c|c|c|c|}
\hline & MRI/ASC & Sensitivity & Specificity & PPV & NPV \\
\hline CFL & $4 / 5$ & $\begin{array}{l}80.0 \% \\
\text { (CI 30-99) }\end{array}$ & $\begin{array}{l}100 \% \\
\text { (CI 83-100) }\end{array}$ & $\begin{array}{l}100 \% \\
\text { (CI 40-100) }\end{array}$ & $\begin{array}{l}96.0 \% \\
\text { (CI 78-100) }\end{array}$ \\
\hline PTFL & $3 / 1$ & $\begin{array}{l}100 \% \\
\text { (CI 5-100) }\end{array}$ & $\begin{array}{l}86.7 \% \\
\text { (CI 76-99) }\end{array}$ & $\begin{array}{l}33.3 \% \\
\text { (CI 2-87) }\end{array}$ & $\begin{array}{l}100 \% \\
\text { (CI 84-100) }\end{array}$ \\
\hline Deltoid lig. & $5 / 0$ & - & $\begin{array}{l}83.3 \% \\
\text { (CI 65-94) }\end{array}$ & $\begin{array}{l}0 \% \\
\text { (CI 0-54) }\end{array}$ & $\begin{array}{l}100 \% \\
\text { (CI 83-100) }\end{array}$ \\
\hline Basset lig. & $1 / 8$ & $\begin{array}{l}0 \\
\text { (CI } 0-40)\end{array}$ & $\begin{array}{l}94.7 \% \\
\text { (CI 75-99) }\end{array}$ & $\begin{array}{l}0 \% \\
\text { (CI 0-95) }\end{array}$ & $\begin{array}{l}71.9 \% \\
\text { (CI 53-87) }\end{array}$ \\
\hline Cervical lig. & $1 / 0$ & - & $\begin{array}{l}96.2 \% \\
\text { (CI 81-99) }\end{array}$ & $\begin{array}{l}0 \% \\
\text { (CI 0-95) }\end{array}$ & $\begin{array}{l}100 \% \\
\text { (CI 85-100) }\end{array}$ \\
\hline Total & $14 / 14$ & $\begin{array}{l}28.4 \% \\
\text { (CI 10-58) }\end{array}$ & $\begin{array}{l}37.8 \% \\
\text { (CI 16-64) }\end{array}$ & $\begin{array}{l}29.3 \% \\
\text { (CI 10-58) }\end{array}$ & $\begin{array}{l}38.4 \% \\
\text { (CI 16-64) }\end{array}$ \\
\hline
\end{tabular}

Reliability of MRI is given by calculating specificity, sensitivity, positive predictive value $(P P V)$, negative predictive value $(N P V)$. All data are shown with $95 \%$ confidence interval (CI), CFL calcaneofibular ligament, PTFL posterior talofibular ligament, lig. ligament 
Table 5 Incidence and classification of cartilage damage in MRI and arthroscopy

\begin{tabular}{llll}
\hline & Outerbridge MRI & $\begin{array}{l}\text { Berndt + Harty } \\
\text { MRI }\end{array}$ & $\begin{array}{l}\text { Outerbridge } \\
\text { arthroscopy }\end{array}$ \\
\hline Grade I & $47.4 \%(n=9)$ & $62.5 \%(n=15)$ & $8.3 \%(n=1)$ \\
Grade II & $21.1 \%(n=4)$ & $37.5 \%(n=9)$ & $8.3 \%(n=1)$ \\
Grade III & $26.3 \%(n=5)$ & & $25.0 \%(n=3)$ \\
Grade IV & $5.3 \%(n=1)$ & & $58.3 \%(n=7)$ \\
TOTAL & 19 & 24 & 12 \\
\hline
\end{tabular}

Images were evaluated using the Outerbridge grading scale [22] (MRI and arthroscopy) and Berndt and Harty classification system [2] (MRI only)

Table 6 Reliability/validity of MRI for cartilage defects

\begin{tabular}{lll}
\hline & Outerbridge classification & $\mathrm{B}+$ H classification \\
\hline Sensitivity & $91.7 \%$ (CI 61.5-99.7) & $91.7 \%$ (CI 61.5-99.7) \\
Specificity & $55.6 \%$ (CI 30.7-78.5) & $27.8 \%$ (CI 9.7-53.5) \\
PPV & $57.9 \%$ (CI 44.4-70.3) & $45.8 \%$ (CI 37.7-54.2) \\
NPV & $90.9 \%$ (CI 59.4-98.6) & $83.3 \%$ (CI 39.9-97.4) \\
Correlation & $r_{\mathrm{s}}=0.62 ; p<0.001$ & $r_{\mathrm{s}}=0.52 ; p=0.003$ \\
\hline
\end{tabular}

Outerbridge grading scale and Berndt and Harty $(\mathrm{B}+\mathrm{H})$ classification system were applied. All values are shown with $95 \%$ confidence interval (CI). Correlation between MRI and arthroscopy was determined by calculating Spearman's rank coefficient $\left(r_{\mathrm{s}}\right)$

$P P V$ positive predictive value; $N P V$ negative predictive value

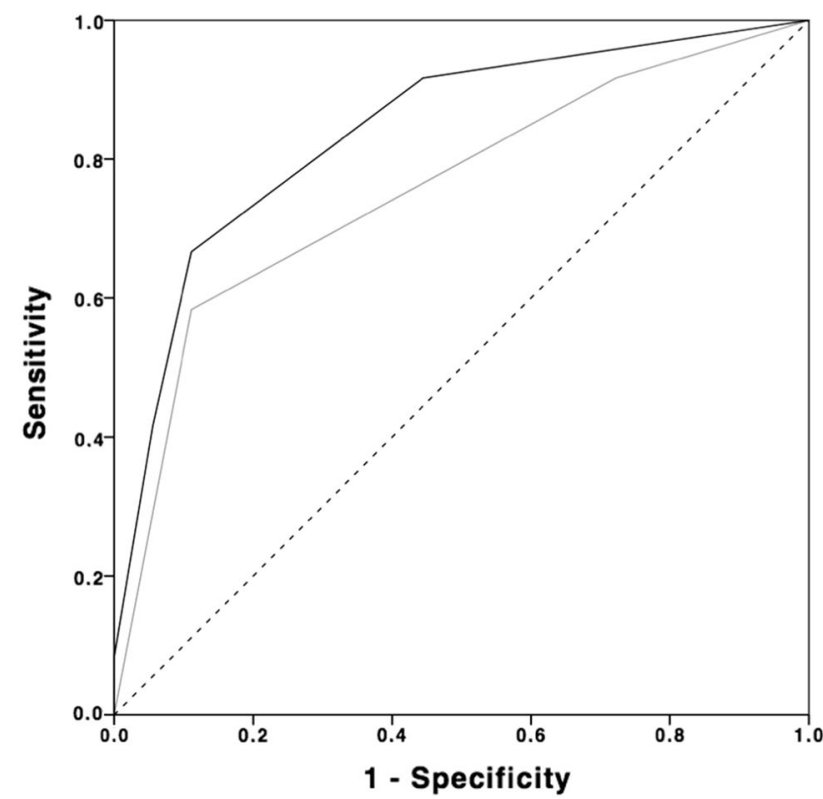

Fig. 2 Accuracy of Outerbridge and Berndt and Harty classification in ankle MRI. Accuracy of Outerbridge grading scale (black) and Berndt and Harty $(\mathrm{B}+\mathrm{H})$ classification system (grey) was evaluated by calculating the area under the receiver operating curve (AUC). Accuracy for the Outerbridge classification system was $84 \%$ ( $p=0.002 ; 95 \%$ CI range $69-99)$ compared to $76 \%(p=0.02 ; 95 \%$ CI range 57-94) for B + H classification system the receiver operating curve (AUC) was $84 \%(p=0.002$; 95\% CI range 69-99) compared to $76 \%$ ( $p=0.021 ; 95 \%$ CI range 57-94) for B + H classification system. Figure 2 shows the AUC for both classification systems.

\section{Discussion}

The most important finding of this present study was that CAI was associated with a high incidence of additional pathologies in both MRI and arthroscopy, albeit with low sensitivity and high specificity, MRI may in some cases deliver underestimated information for present comorbidities. Therefore, standard morphological MRI may be a helpful tool for preoperative evaluation, but it cannot replace arthroscopy as gold standard for the diagnosis of comorbidities in CAI. MRI was most accurate for the detection of anterolateral impingement. These results are comparable to the findings from Clanton et al. for diffuse synovitis, albeit they could not detect any specificity for diffuse synovitis [7]. Even though we also gathered satisfying results for peroneal tendionpathy, we cannot approve this result unconditionally due to the low prevalence of peroneal lesions in our cohort. O'Neill et al. [21] conducted a study in which they also found that even though MRI delivers reliable results, the detection rate of additional lesions (chondral, loose bodies, peroneal tendon ruptures) in CAI patients ranged from 40 to $89 \%$. Our data suggest that negative MRI results should be handled cautiously, especially regarding loose bodies. Only half of the loose bodies found intraoperatively were detected preoperatively through MRI. These results support the findings of O'Neill et al. [21] in which only 57\% of loose bodies could be identified through MRI. In contrast, MRI showed a high sensitivity but a relatively low specificity and positive predictive values for cartilage defects. Gatlin et al. [9] found roughly similar results for preoperative MRI detecting cartilage defects of the talus in patients undergoing ankle surgery. Furthermore our results indicate that using the Outerbridge classification system seems slightly more accurate than using the classification system proposed by Berndt and Harty (Fig. 2). Superiority of the Outerbridge system may be caused by the possibility of applying this system intraoperatively by macroscopic examination of the cartilage.

With regard to a high incidence of comorbidities found in MRI and arthroscopically, our results confirm the findings of Choi et al. [6] and Kibler [17] in which they detected comorbidities in $96.9 \%$, respectively, in $83 \%$ of observed patients who underwent lateral ligament reconstruction.

All observed pathologies showed only a low to moderate correlation between preoperative MRI and arthroscopy. 
Arthroscopy fully agreed in four cases with additional ligament lesions only with the results of preoperative MRI. All of these patients showed lesions of the lateral ligament complex (CFL and/or PTFL). All other arthroscopically accounted ligamentous pathologies $(n=8)$ were a hypertrophic Basset ligament. MRI revealed five pathologic changes in the deltoid ligament, which could not be confirmed by the data gathered from arthroscopy. But since only patients with lateral ankle instability were included in this study there may have been some alterations in the ligaments of the medial complex that were not clinically present. Similar findings were reported by Cha et al., in which they also found low sensitivity for additional ligament pathologies [4].

This study represents several limitations:

First, retrospective analyses of arthroscopic images rely on the intraoperatively collected data and the experience of the reader. Both surgeons involved in the study are specialized in foot and ankle surgery. Although, a discrepancy in the experience between the readers was present (resident and experienced surgeon), a good to excellent inter- and intrarater reliability could be achieved. Therefore, this irregularity seems to be negligible. Second, due to the low number of patients, incidences may get over- or underestimated, and therefore, occurring differences between MRI and arthroscopy may appear more substantial. Third, the time between MRI and arthroscopy was prolonged in some cases. Thus, pathologies found in arthroscopy may not have been present during the time of MRI. But the mean time of 3 months from MRI to arthroscopy seems to be in an acceptable range.

\section{Conclusion}

This study shows that CAI is associated with a high incidence of additional pathologies. For some comorbidities, MRI delivers low sensitivity and weak to moderate correlation to arthroscopic findings. Therefore, especially negative results in MRI should be handled with caution. MRI has shown to be a helpful tool for preoperative evaluation of additional lesions in patients with CAI. But diagnostic arthroscopy remains gold standard especially if a discrepancy between radiological and clinical findings is present.

\section{Compliance with ethical standards}

Conflict of interest The authors can declare that there are no existing conflicts of interest.

Sources of funding No external funding was provided for the study.
Ethical approval This study was approved by the local ethics committee of the Medical University of Vienna (EK No. 1072/2016).

Informed consent For this type of study informed consent is not necessary.

Open Access This article is distributed under the terms of the Creative Commons Attribution 4.0 International License (http://creativecommons.org/licenses/by/4.0/), which permits unrestricted use, distribution, and reproduction in any medium, provided you give appropriate credit to the original author(s) and the source, provide a link to the Creative Commons license, and indicate if changes were made.

\section{References}

1. Alparslan L, Chiodo C (2008) Lateral ankle instability: MR imaging of associated injuries and surgical treatment procedures. Semin Musculoskelet Radiol 12:346-358

2. Berndt AL, Harty M (1959) Transchondral fractures (osteochondritis dissecans) of the talus. J Bone Joint Surg Am 41(A):988-1020

3. Broström L (1966) Sprained ankles. VI. Surgical treatment of "chronic" ligament ruptures. Acta Chir Scand 132:551-565

4. Do Cha S, Kim HS, Chung ST, Yoo JH, Park JH, Kim JH, Hyung JW (2012) Intra-articular lesions in chronic lateral ankle instability: comparison of arthroscopy with magnetic resonance imaging findings. Clin Orthop Surg 4:293-299

5. Cho B-K, Kim Y-M, Kim D-S, Choi E-S, Shon H-C, Park K-J (2012) Comparison between suture anchor and transosseous suture for the modified-Broström procedure. Foot Ankle Int 33:462-468

6. Choi WJ, Lee JW, Han SH, Kim BS, Lee SK (2008) Chronic lateral ankle instability: the effect of intra-articular lesions on clinical outcome. Am J Sports Med 36:2167-2172

7. Clanton TO, Ho CP, Williams BT, Surowiec RK, Gatlin CC, Haytmanek CT, LaPrade RF (2016) Magnetic resonance imaging characterization of individual ankle syndesmosis structures in asymptomatic and surgically treated cohorts. Knee Surg Sports Traumatol Arthrosc 24:2089-2102

8. DIGiovanni BF, Fraga CJ, Cohen BE, Shereff MJ (2000) Associated injuries found in chronic lateral ankle instability. Foot Ankle Int 21:809-815

9. Gatlin CC, Matheny LM, Ho CP, Johnson NS, Clanton TO (2015) Diagnostic accuracy of 3.0 Tesla magnetic resonance imaging for the detection of articular cartilage lesions of the talus. Foot Ankle Int 36:288-292

10. Guillo S, Bauer T, Lee JW, Takao M, Kong SW, Stone JW, Mangone PG, Molloy A, Perera A, Pearce CJ, Michels F, Tourné Y, Ghorbani A, Calder J (2013) Consensus in chronic ankle instability: aetiology, assessment, surgical indications and place for arthroscopy. Orthop Traumatol Surg Res 99:411-419

11. Hintermann B, Boss A, Schäfer D (2002) Arthroscopic findings in patients with chronic ankle instability. Am J Sports Med 30:402-409

12. Jackson W, McGarvey W (2006) Update on the treatment of chronic ankle instability and syndesmotic injuries. Curr Opin Orthop 17:97-102

13. Jordan LK, Helms CA, Cooperman AE, Speer KP (2000) Magnetic resonance imaging findings in anterolateral impingement of the ankle. Skeletal Radiol 29:34-39 
14. Joshy S, Abdulkadir U, Chaganti S, Sullivan B, Hariharan K (2010) Accuracy of MRI scan in the diagnosis of ligamentous and chondral pathology in the ankle. Foot Ankle Surg 16:78-80

15. Keller M, Grossman J, Caron M, Mendicino RW (1996) Lateral ankle instability and the Brostrom-Gould procedure. J Foot Ankle Surg 35:513-520

16. Kerkhoffs GMMJ, Kennedy JG, Calder JDF, Karlsson J (2016) There is no simple lateral ankle sprain. Knee Surg Sports Traumatol Arthrosc 24:941-943

17. Kibler WB (1996) Arthroscopic findings in ankle ligament reconstruction. Clin Sports Med 15:799-804

18. Lee M, Kwon JW, Choi WJ, Lee JW (2015) Comparison of outcomes for osteochondral lesions of the talus with and without chronic lateral ankle instability. Foot Ankle Int 36:1050-1057

19. Linklater J (2009) MR imaging of ankle impingement lesions. Magn Reson Imaging Clin N Am 17:775-800

20. Matsui K, Burgesson B, Takao M, Stone J, Guillo S, Glazebrook M, Group EAAI (2016) Minimally invasive surgical treatment for chronic ankle instability: a systematic review. Knee Surg Sport Traumatol Arthrosc 24:1040-1048
21. O’Neill PJ, Van Aman SE, Guyton GP (2009) Is MRI adequate to detect lesions in patients with ankle instability? Clin Orthop Relat Res 468:1115-1119

22. Outerbridge RE (1961) The etiology of chondromalacia patellae. J Bone Joint Surg Br 43(B):752-757

23. Rodriguez-Merchan EC (2012) Chronic ankle instability: diagnosis and treatment. Knee Surg Sports Traumatol Arthrosc 132:211-219

24. Strauss JE, Forsberg JA, Lippert FG (2007) Chronic lateral ankle instability and associated conditions: a rationale for treatment. Foot Ankle Int 28:1041-1044

25. Takao M, Uchio Y, Naito K, Fukazawa I, Ochi M (2005) Arthroscopic assessment for intra-articular disorders in residual ankle disability after sprain. Am J Sports Med 33:686-692

26. Walther M, Kriegelstein S, Altenberger S, Volkering C, Röser A, Wölfel R (2013) Lateral ligament injuries of the ankle joint. Unfallchirurg 116:776-780

27. Watson AD (2007) Ankle instability and impingement. Foot Ankle Clin 12:177-195 